\title{
ESTUDIOS CROMOSÓMICOS EN ARACHIS (LEGUMINOSAE) ${ }^{1}$
}

\author{
por GRACIELA INES LAVIA²
}

\section{Summary}

\begin{abstract}
Chromosome numbers are reported for 38 accesions representing 17 species of five sections of genus Arachis. First chromosome counts are reported for the following 8 species: Seci. Extranervosae: A. retusa, Sect. Heteranthae: A. Giacomettii, Sect. Procumbentes: A. : /allsii, Sect. Arachis: A. decora, A. microsperma, A. palustris, A. trinitensis and A.Williamsii. Previous reports of nine species are confirmed. All the studied species have $2 n=2 x=20$, except one accession of $A$. palustris, that has $2 n=2 x=18$, which represents probably a new basic number $\mathrm{x}=9$ for the genus. Satellited chromosomes are analized for most species. "A" chromosomes are found only in A. microsperma and A. trinitensis (Sect. Arachis).
\end{abstract}

\section{Introducción}

El género Arachis, originario de Sudamérica, cuenta con 69 especies agrupadas en 9 secciones: Trierectoides, Erectoides, Extranervosae, Triseminatae, Heteranthae, Caulorrhizae, Procumbentes, Rhizomatosae y Arachis (Krapovickas y Gregory, 1994), distribuidas al este de la Cordillera de los Andes, desde el río Amazonas hasta el río de La Plata.

Husted $(1933,1936)$ fue el primero en estudiar la morfología de los cromosomas de Arachis. Diferenció en el complemento 2 pares, un par con constricción secundaria o cromosomas SAT y otro par de cromosomas pequeños a ios que llamó cromosomas " $\mathrm{A}$ ".

La mayoría de las especies son diploides $(2 n=2 x=20)$ y sólo cuatro son tetraploides $(2 n=4 x=40)$; dos de las especies tetraploides, $A$. monticola (Krapovickas y Rigoni, 1951 y 1957) y A. hypogaea pertenecen a la sección Arachis y las otras dos, A. glabrata (Gregory et al., 1973) y

\footnotetext{
1 Este trabajo forma parte de la tesis doctoral a ser presentada en la Facultad de Ciencias Exactas, Físicas y Naturales de la Universidad Nacional de Córdoba.

? Becaria del CONICET, Instituto de Botánica del Nordeste. C.C. 209, 3400 Corrientes, Argentina.
}

A. pseudovillosa (Fernández y Krapovickas, 1994), pertenecen a la sección Rhizomatosae.

Fernández y Krapovickas (1994) describieron los cromosomas somáticos de 40 especies, estableciendo diferentes tipos de cromosomas SAT. La sección Arachis fue la única en la que se observaron cromosomas " $\mathrm{A}$ ".

En el presente trabajo se dan a conocer los primeros recuentos cromosómicos efectuados en 8 especies pertenecientes a 4 secciones y se confirman los números en otras 9. Además se analizan los cromosomas SAT y la ocurrencia de cromosomas " $\mathrm{A}$ ".

\section{Material y Métodos}

Los testigos de las especies estudiadas están depositados en el Herbario del Instituto de Botánica del Nordeste (CTES) y en el Centro Nacional de Recursos Genéticos (CEN) de Brasilia. La lista del material estudiado figura en ia Tabla 1.

La técnica citológica utilizada ha sido descripta detalladamente por Fernández y Krapovickas (1994). Las raíces fueron pretratadas durante 3 hs en 8-hidroxiquinoleína $0.002 \mathrm{M}$ a temperatura ambiente, fijadas en $5: 1$ alcohol absoluto : ácido láctico a $4^{\circ} \mathrm{C}$ y luego conservadas en etanol $70 \%$. La tinción fue realizada mediante la técnica de Feulgen. 
Tabla 1. Material estudiado y cromosomas.

\begin{tabular}{|c|c|c|c|c|c|}
\hline ESPECIISS & $2 n$ & FIG. & COLECTOR Y LOCALIDAD & $\begin{array}{l}\text { CROM. } \\
\text { A }\end{array}$ & $\begin{array}{l}\text { TIPO } \\
\text { SAT }\end{array}$ \\
\hline \multicolumn{6}{|l|}{ Sect. Extranervosae } \\
\hline \multirow{10}{*}{$\begin{array}{l}\text { A. Burchellii Krapov. \& } \\
\text { W.C. Gregory }\end{array}$} & 20 & $1 \mathrm{~A}$ & VKRSv 6556. Brasil, TO, Mun. Colinas do & & \\
\hline & & & Tocantins, $3 \mathrm{~km} S$ de Araguaina, BR- 153 . & - & 6 \\
\hline & 20 & & VSSaGdW 7805. Brasil, MT, $16,4 \mathrm{~km}$ W de & & \\
\hline & & & São Felix do Araguaia, para a BR-158. & - & \\
\hline & 20 & & VSSaGdW 7821. Brasil, MT, Mun. Luciara, & & \\
\hline & & & $14 \mathrm{~km} \mathrm{~S}$ de Porto Alegre do Norte. & - & 3 \\
\hline & 20 & & VSSaGdW 7862.Brasil, MT, Santa Teresinha. & - & \\
\hline & 20 & & VVeSv 8359. Brasil, MA, Mun. Montes Altos, & & \\
\hline & & & $17 \mathrm{~km} \mathrm{~N}$ de Carolina. & - & \\
\hline & 20 & & VVeSv 8405. Brasil, MA, $6 \mathrm{~km}$ NE de Balsas. & - & \\
\hline \multirow{2}{*}{$\begin{array}{l}\text { A. Macedoi Krapov. \& } \\
\text { W.C. Gregory }\end{array}$} & 20 & & VKRSv 6609. Brasil, MA, 11,8 km E de Carolina. & a. - & 3 \\
\hline & 20 & $1 \mathrm{~B}$ & $\begin{array}{l}\text { VR 7533. Brasil, MG, Capinopolis, Faz. } \\
\text { Santa Terezinha. }\end{array}$ & - & 3 \\
\hline A. aff. Pietrarellii Krapov.\& & 20 & $1 C$ & VSSaGdW 7861. Brasil, MT, Mun. Santa & & \\
\hline W.C. Gregory & & & Teresinha: Santa Teresinha. & - & 3 \\
\hline A. prostrata Benth. & 20 & & VPmSv 13040. Brasil, MA, Mun. Balsas. & - & \\
\hline \multirow{5}{*}{$\begin{array}{l}\text { * Aetusa Krapov., W.C. } \\
\text { Gregory \& Valls }\end{array}$} & 20 & 1D & VSW 9950. Brasil, GO, $2 \mathrm{~km}$ ao norte de & & \\
\hline & & & Teresinha e $34 \mathrm{~km}$ ao sul do rio Paranā, ao & & \\
\hline & & & longo da estrada para Monte Alegre, $590 \mathrm{~m}$. & - & 3 \\
\hline & 20 & & VPmSv 12939. Brasil, TO, Mun. Paranã, & & \\
\hline & & & 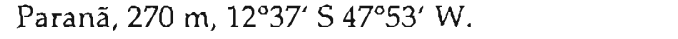 & - & \\
\hline \multirow[t]{2}{*}{ A. villosulicarpa Hoehne } & 20 & & $\begin{array}{l}\text { VKSSv 8816. Brasil, MT, Aldeia Aroeira } \\
\text { (cerca de Vilhena). }\end{array}$ & - & 3 \\
\hline & $20(40)$ & ) $1 \mathrm{E}$ & $\begin{array}{l}\text { VKSSv 8818. Brasil, MT, Aldeia Aroeira } \\
\text { (cerca de Vilhena). }\end{array}$ & - & 3 \\
\hline \multirow{5}{*}{$\begin{array}{l}\text { Sect. Heteranthae } \\
\text { * A. Giacomettii Krapov., } \\
\text { W.C. Gregory, Valls \& } \\
\text { C.E. Simpson }\end{array}$} & & & & & \\
\hline & 20 & & $\begin{array}{l}\text { VPzVaW 13202. Brasil, MG, Mun. } \\
\text { Montalvania, 8,5 km NW de Montalvania, } \\
\text { na estrada para Pitarana. }\end{array}$ & - & \\
\hline & 20 & $1 \mathrm{~F}$ & WPn 201. Brasil, MG, 8,5 km NW de & & \\
\hline & & & Montalvania, na estrada para Pitarana. & - & $10 ?$ \\
\hline & 20 & & $\begin{array}{l}\text { WPn 178. Brasil, MG, } 12 \mathrm{~km} \text { de Montalvania, } \\
\text { desde o rio Coxá, em direção a Pitarana. }\end{array}$ & - & \\
\hline \multirow{7}{*}{$\begin{array}{l}\text { A. sylvestris (A. Chev.) } \\
\text { A. Chev. }\end{array}$} & 20 & $1 G$ & VVeSv 8373. Brasil, MA, 9,5 km ao $\mathrm{N}$ de & & \\
\hline & & & Carolina, na estrada para Estreito. & - & 10 \\
\hline & $20(40)$ & $1 \mathrm{H}$ & $\begin{array}{l}\text { VVeSv 8386. Brasil, MA, } 60 \mathrm{~km} \text { E de Carolina, } \\
\text { BR-230. }\end{array}$ & - & 10 \\
\hline & 20 & & VVeSv 8435. Brasil, MA, 70 km NE de Sāo & & \\
\hline & & & Raimundo, BR-230. & - & 10 \\
\hline & 20 & & VVeSv 8520. Brasil, PI, Mun. Oeiras, 35 km & & \\
\hline & & & NE de Cristino Castro, BR-135. & - & 10 \\
\hline \multicolumn{6}{|l|}{ Sect. Procumbentes } \\
\hline $\begin{array}{l}\text { * A. Vallsi Krapov. \& } \\
\text { W.C. Gregory }\end{array}$ & 20 & $2 \mathrm{~A}$ & $\begin{array}{l}\text { VRGeSv 7635. Brasil, MS, } 37,8 \mathrm{~km} \text { a oeste da } \\
\text { saida de Miranda na BR-262. }\end{array}$ & - & \\
\hline \multicolumn{6}{|l|}{ Sect. Rhizomatosae } \\
\hline A. glabrata Benth. & 40 & & $\begin{array}{l}\text { Ab 6115. Paraguay, Dep. Misiones, } 7 \mathrm{~km} \mathrm{E} \\
\text { de San Ignacio camino a Santa Rosa. }\end{array}$ & & \\
\hline
\end{tabular}




\section{Sect. Arachis}

* A. decora Krapov., W.C. 20 Gregory \& Valls

* A. microsperma Krapov., W.C. Gregory \& Valls

* A. palustris Krapov., W.C. Gregory \& Valls A. stenospermi Krapov. \& W.C. Gregory

A.trinitensis Krapov. \&
W.C. Gregory
A. villosa Benth.
* A. Williamsii Krapov. \&
W.C. Gregory

$2 B$ VI'mSv 12893. Brasil, TO, Mur. Combinado, $12^{\circ} 55^{\prime} \mathrm{S}, 46^{\circ} 35^{\prime} \mathrm{W}$. VSPmPzRs 13290. Brasil, GO, Mun. Monte Alegre de Goiás, $13^{\circ} 18^{\prime} \mathrm{S}, 46^{\circ} 42^{\prime} \mathrm{W}$.

$202 C \quad$ VRGeSv 7681=13545. Brasil, MS, Mun. Bela Vista, area urbanizada proxima a margem do rio Apa.

18 2D VPmSv 13023. Brasil, TO, Mun. Filadelfia, $7^{\circ} 25^{\prime} \mathrm{S}, 43^{\circ} 37^{\circ} \mathrm{W}$.

2E VSSv 7382. Brasil, SP, Mun. São Sebastião, Pontal da Olaria. VMiSv 10229. Brasil, SP, Mun. Cananeia, Carraneia.

VSSaGdW 7762. Brasil, MT, Mun. Araguaiana, km 38 da BR-158, Barra do Garças-Nova Xavantuna, $4.50 \mathrm{~m}$.

VKSSv 9017. Brasil, MT, 6,6 km W do rio Bambá, BR-364.

2I: VSv 10309. Brasil, MT, Mun. Rondonópolis, BR-364, ca. $200 \mathrm{~m}$ W do rio Vermelho e $300 \mathrm{~m}$ $W$ do acceso a Rondonópolis.

VGaRoSv 12488. Brasil, MT, Araguaıana $15^{\circ} 34^{\circ} \mathrm{S}, 52^{\circ} 13^{\prime} \mathrm{W}$.

VGaRoSv 12575. Brasil, MT, General

Carneiro $15^{\circ} 41^{\prime} \mathrm{S}, 52^{\circ} 46^{\circ} \mathrm{W}$.

VGaSv 12646. Brasil, MT, Santo Antonio do Leverger $15^{\circ} 43^{\prime} \mathrm{S}, 55^{\circ} 42^{\prime} \mathrm{W}$.

WiCl 1117. Bolivia, Dep. Beni, Trinidad, Universidad Técnica del Beni, 20 m NE del edificio laboratorios.

La 17. Argentina, Entre Ríos, Dep. Colón, Colón, zona del puerto.

$+2$

$+3$

$+5$

$+$$$
-5
$$$$
+5
$$$$
+5
$$$$
+5
$$

2H WiCl 1118. Bolıvia, Dep. Beri, Trinidad, Universidad Técnica del Beni, $15 \mathrm{~m}$ E del edificio laboratorios.

* primer recuento para la especie.

(40) células polisomáticas.

Ab M.M. Arbo, $\mathrm{Cl}=\mathrm{D}$. Claure, Ga=M.L. Galgaro, Ge=M.A.N. Gerin, Gd I.J. de Godoy, K: A. Krapovickas, $\mathrm{L} a=$ G.I. Lavia, Mi=S. Miotto, Pm=R.N. Pittman, $\mathrm{P} n=P$. Pinheiro, Pz=E.A. Pizarro, R=V.R. Rao, Ro=D.M.S. Rocha, Rs=R.C. dos Santos, S=C.E. Simpson, Sa=H.T. Stalker, Sv : G.P. Silva, V J.F.M. Valls, Va S.E.S. Valente, Ve=R.F. de Arruda Veiga, W=W.L. Werneck, Wi=D.F. Williams.

\section{Resultados}

En la Tabla 1 se muestran los números cromosómicos, los tipos de cromosomas SAT y la presencia o no de los cromosomas "A" en las distintas especies analizadas. Las especies marcadas con un asterisco $\left(^{*}\right)$ corresponden a nuevos recuentos.
En este trabajo se presentan por primera vez los números cromosómicos de las siguientes especies: Secc. Extranervosae: A. retusa (9950, 12939), Secc. Heteranthae: A. Giacomettii (13202, 178, 201), Secc. Procumbentes: A. Vallsii (7635), Secc. Arachis: A. decora (12893, 13290), A. microsperma (7681), A. palustris (13023), A. trinitensis (1117) y A. Williamsii (11.18), todas 
ellas con $2 n=2 x=20$, excepto $A$. palustris que posee $2 n=2 x=18$ (Fig. 2D).

Por otro lado se confirman los números de 9 especies (26 accesiones): Secc. Extranervosae: $A$. Burchellii (6556, 7805, 7821, 7862, 8359, 8405), A. Macedoi $(6609,7533)$, A. Pietrarellii (7861), A. prostrata (13040) y A. villosulicarpa $(8816,8818)$; Secc. Heteranthae: A. sylvestris (8373, 8386, 8435, 8520); Secc. Rhizomatosae: A. glabrata (6115); Secc. Arachis: A. stenosperma (7382, 7762, 9017, $10229,10309,12488,12575,12646)$ y A. villosa (17), todas dipioides, excepto A. glabrata, que es tetraploide.

Se observaron células polisomáticas en $A$. sylvestris (Fig. $1 \mathrm{H})$, A. villosulicarpa y $A$ stenosperma (Fig. 2G).

En $A$. decora, A. palustris y A. Williamsii, pertenecier:tes a la sección Arachis, no se observaron cromosomas "A"; por lo tanto, hasta el momento son 10 las especies que carecen de este par en dicha sección.

Los cromosomas SAT fueron identificados de acuerdo con la clasificación establecida por Fernández y Krapovickas (1994), quienes determinaron 10 tipos diferentes teniendo en cuenta ei tamaño del satélite y la posición del centrómero.

En especies de la sección Extranervosae se observaron cromosomas SAT de los tipos 3 y 6 , siendo el tipo 3 el más común, hallado en 5 de las 6 especies estudiadas; el tipo 6 fue observado en sólo una accesión de A. Burchellii (Fig. 1A). En lo que respecta a $A$. villosulicarpa, en las dos accesiones estudiadas se hallaron cromosomas SAT del tipo 3, novedad para la especie, ya que Fernández y Krapovickas (1994) sclo hallaron cromosomas SAT de los tipos 1 y 2

Dentro de la sección Heteranthae las 4 accesiones analizadas de $A$. sylvestris presentan cromosomas SAT punctiformes del tipo 10; mientras que en A. Giacomettii (201) se observó un par de cromosomas con satélite muy peque. ño, pero no se pudo establecer si pertenecía ai tipo 9 ó 10.

En especies de la sección Arachis encontramos cromosomas SAT tipos 3, 5 y 6; A. palustris y A. villosa poseen el tipo 3, A. Williamsii el tipo 6.

En A. stenosperma se analizaron 8 accesiones (Tabla 1), 2 provenientes de la costa atlán- tica (Estado de San Pablo) y 6 del Mato Grosso, que presentaron cromosomas SAT de los tipos 3 y 5 respectivamente (Fig. $2 \mathrm{E}$ y $2 \mathrm{~F}$ ).

\section{Discusión y Conclusiones}

Todas las especies del género Arachis estudiadas previamente se caracterizan por el número básico $x=10$, hallándose especies diploides $2 n=2 x=20$ y, en menor frecuencia, tetraploides $2 n=4 x=40$.

En este trabajo se presenta un nuevo número cromosómico para el género $(2 n=2 x=18)$ hallado en cinco plantas de $A$. palustris (Fig. 2D). Probablemente se trate de un nuevo número básico $x=9$, derivado por aneuploidía del $x=10$. Sería necesario analizar mitótica y meióticamente más accesiones de dicha especie, para ver si se repite este número. Cabe señalar que $A$. palustris es la especie silvestre de la sección Arachis que vive más al norte en Brasil (Fig. 5), en suelos totalmente anegados al menos durante una gran parte de su período de desarrollo (Krapovickas y Gregory, 1994).

Fernández y Krapovickas (1994) presentaron una probable evolución estructural de los tipos de cromosomas SAT, donde del tipo 2 derivarían los tipos 1 y 3 , y de éste último los tipos 5 y 6 , entre otros. Los resultados hallados en A. Burchellii, A. villosulicarpa y A. stenosperma apoyan las relaciones propuestas por estos autores. En A. Burchellii se encontraron dos tipos de cromosomas SAT en diferentes accesiones: 3 (7821) y 6 (6556); en A. villosulicarpa Ios cromosomas SAT tipo 3 hallados en las dos accesiones estarían en relación con el tipo 2; en A. stenosperma se hallaron los tipos 5 y 3 .

Cromosomas SAT del tipo 3 son los más frecuentes, ya que han sido observados en un $42 \%$ de las especies estudiadas hasta el momento. Debido a su presencia en un elevado número de especies y secciones, Fernández y Krapovickas (1994) lo propusieron como uno de los cromosomas SAT más primitivos. Los datos obtenidos en este trabajo amplían su área de distribución hacia el centro y norte de Brasil (Fig. 3), siendo de los 10 tipos de cromosomas SAT el de distribución geográfica más amplia.

De las 26 especies silvestres comprendidas en la sección Arachis (Krapovickas y Gregory, 

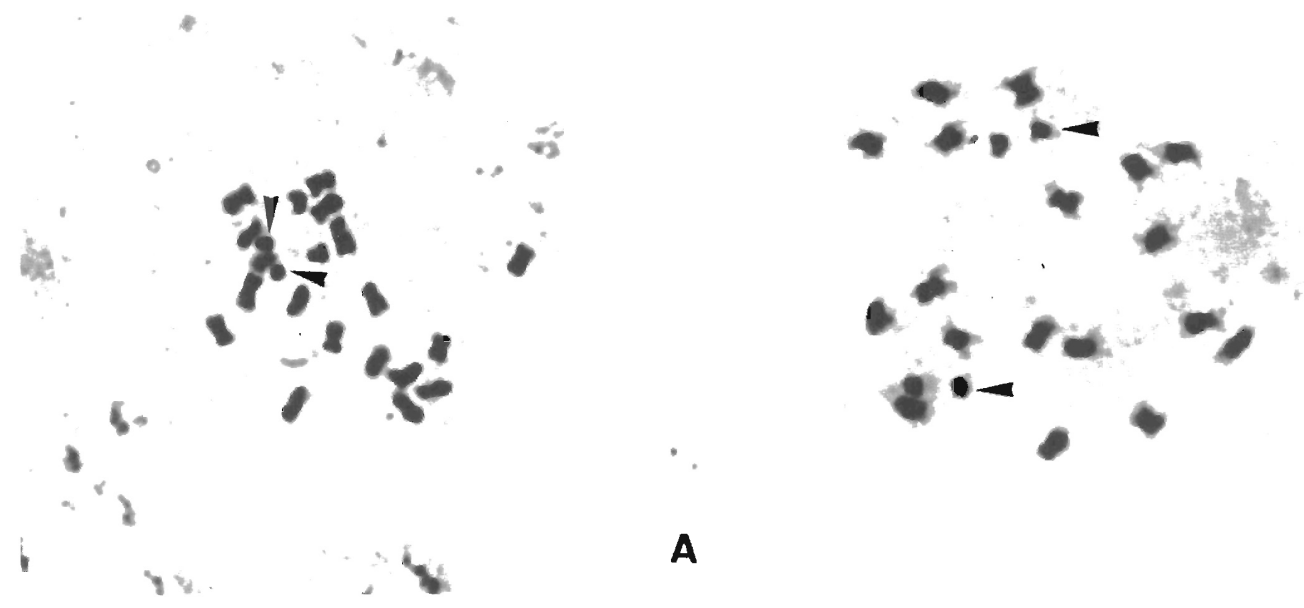

A

B
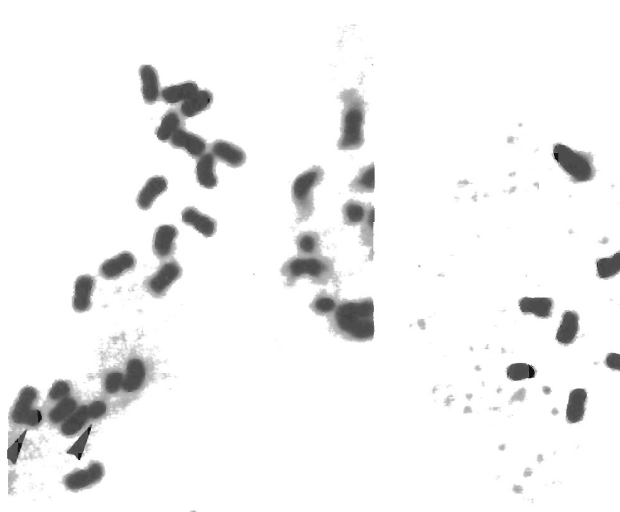

C

D

$\bullet$

$E$

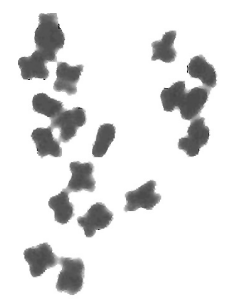

4
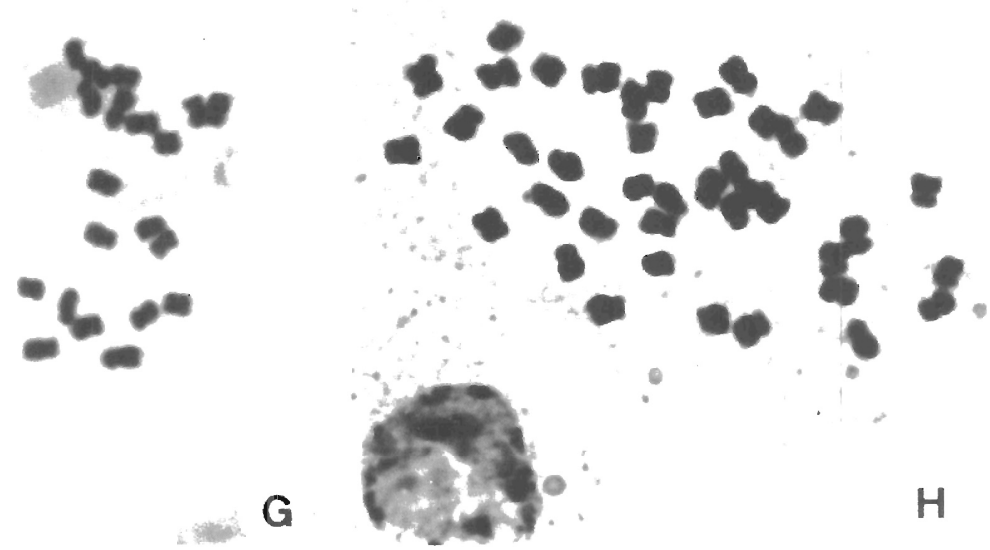

Fig. 1. Cromosomas somáticos de Arachis. A, A. Burchellii, metafase, $2 \mathrm{n}=20$ (6556); B, A. Macedoi, profase, 2n=20 (7533) C, A. Pietrarellii, metafase, $2 \mathrm{n}=20(7861) ; \mathrm{D}$, A. retusa, metafase, $2 \mathrm{n}=20(9950) ; \mathrm{E}$, A. villosulicarpa, metafase, $2 \mathrm{n}=20(8818) ;$ $\mathrm{F}$, A. Giacomettii, metafase, $2 \mathrm{n}=20(201) ; \mathrm{G}-\mathrm{H}_{\mathrm{i}}$, A. sylvestris, $\mathrm{G}$, metafase, $2 \mathrm{n}=20$ (8373); $\mathrm{F}_{\mathrm{i}}$, metafase, célula polisomática, $2 n=40(8386) . X 1500$. sátelite. 


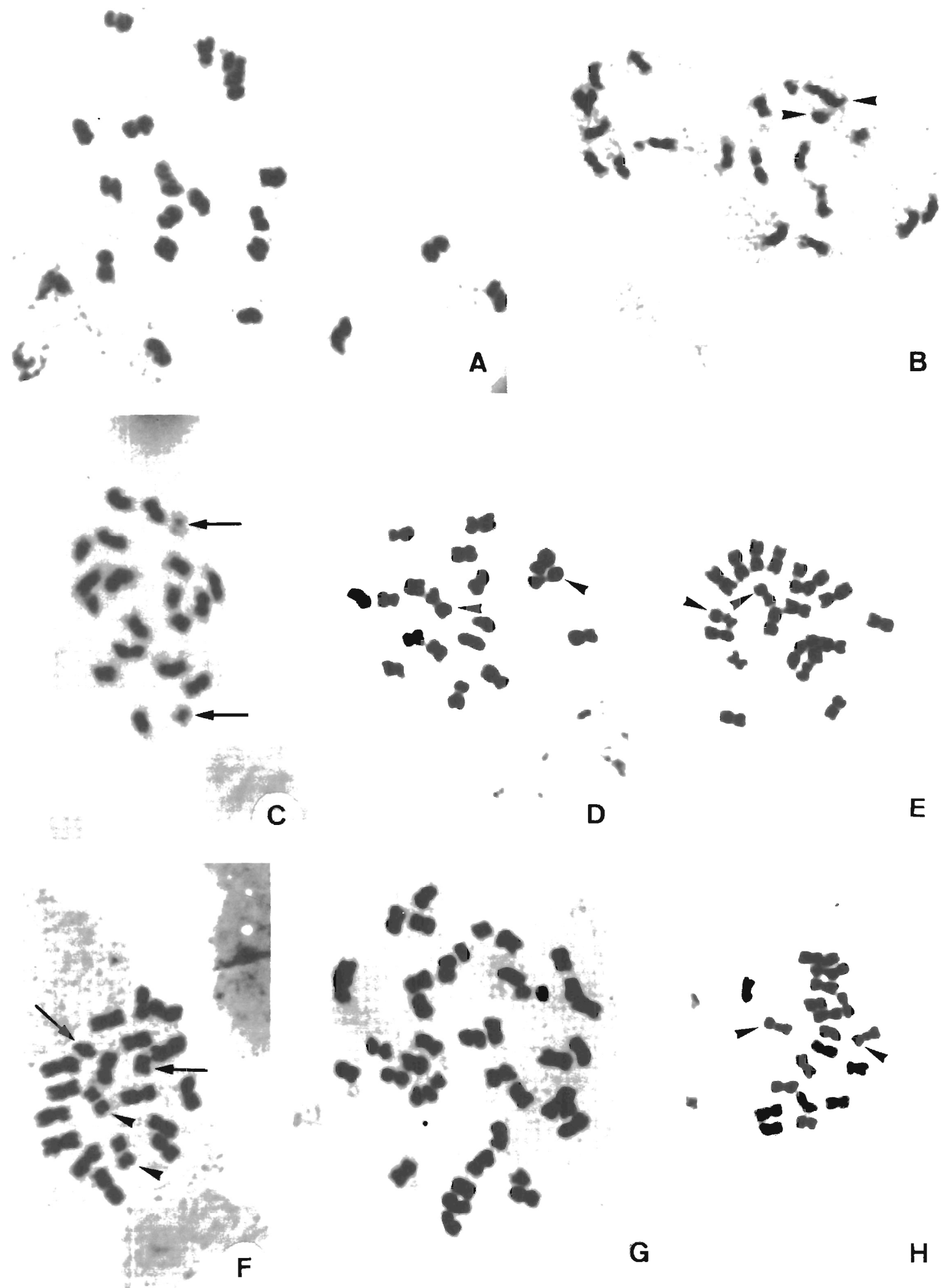

Fig. 2. Cromosomas somáticos de Arachis. A, A. Vallsii, metafase, $2 \mathrm{n}=20 ; \mathrm{B}$, A. decora, profase, $2 \mathrm{n}=20$ (12893); $\mathrm{C}, A$. microsperma, profase, $2 \mathrm{n}=20 ; \mathrm{D}$, A palustris, metafase, $2 \mathrm{n}=18(13023) ; \mathrm{E}-\mathrm{G}$, A.stenosperma, metafases, $\mathrm{E}, 2 \mathrm{n}=20(7382), \mathrm{F}$, $2 \mathrm{n}=20$ (10309), G, célula polisomática, $2 \mathrm{n}=40$ (12575); H, A. Williamsii, metafase, $2 \mathrm{n}=20$. X 1500. sátelite, $\rightarrow$ cromosomas "A" 


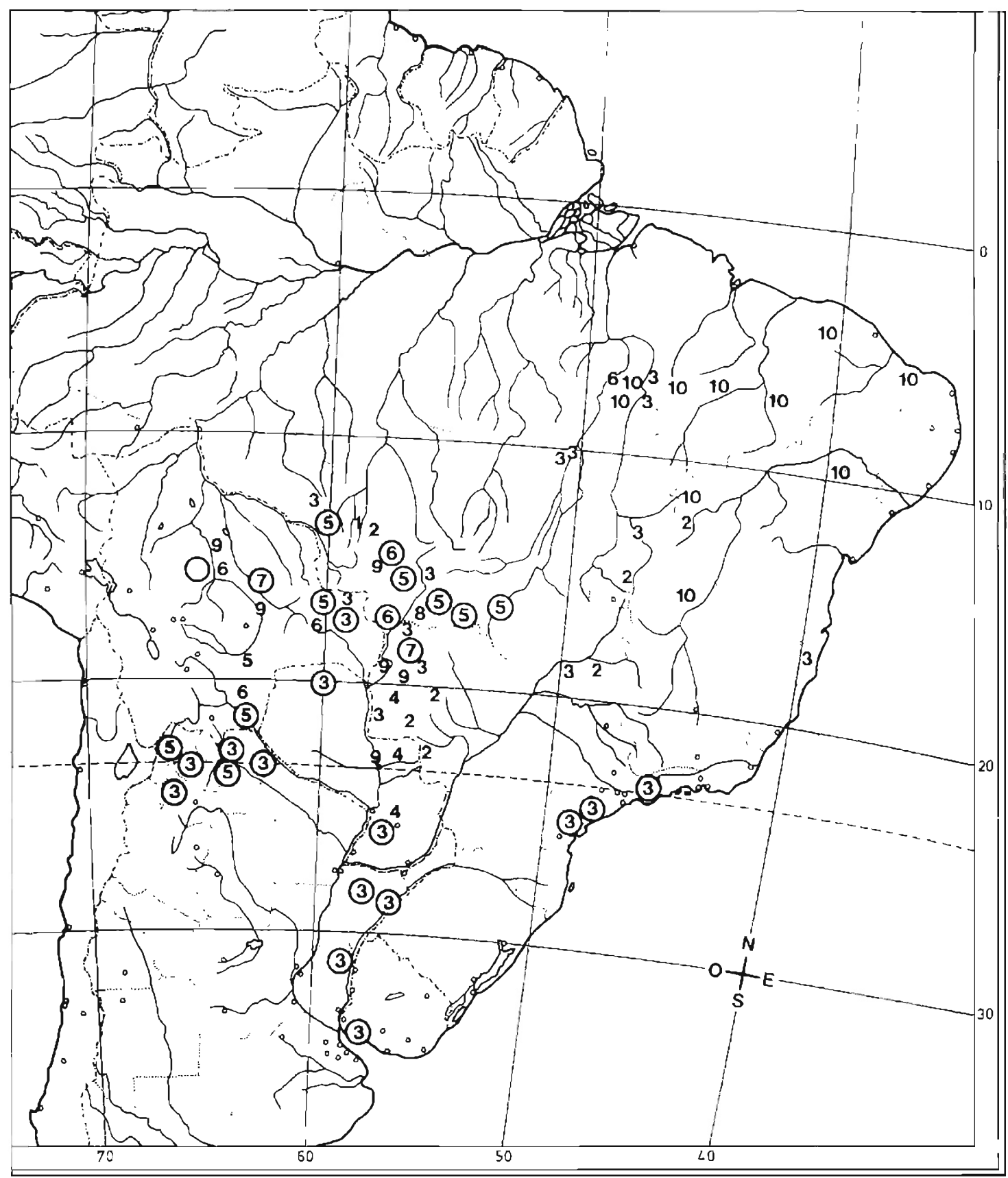

Fig. 3. Distribución geográfica de los cromosomas "A" (O) y SAT (tipos 1 a 10) en el género Arachis. Basado en Fernández y Krapovickas (1994, Fig. 9) más datos propios.

1994), 12 poseen cromosomas " $A$ ", 10 no lo presentan, y de las 4 restantes aún no se tienen datos. En las figuras 4 y 5 se muestra la distribución geográfica de estas especies, observán- dose que todas las que se hallan al este del eje constituido por los ríos Paraguay y Uruguay (58 long. $W$ ) no poseen cromosomas " $\mathrm{A}$ ", excepto $A$. stenosperma, que debido posiblemente 


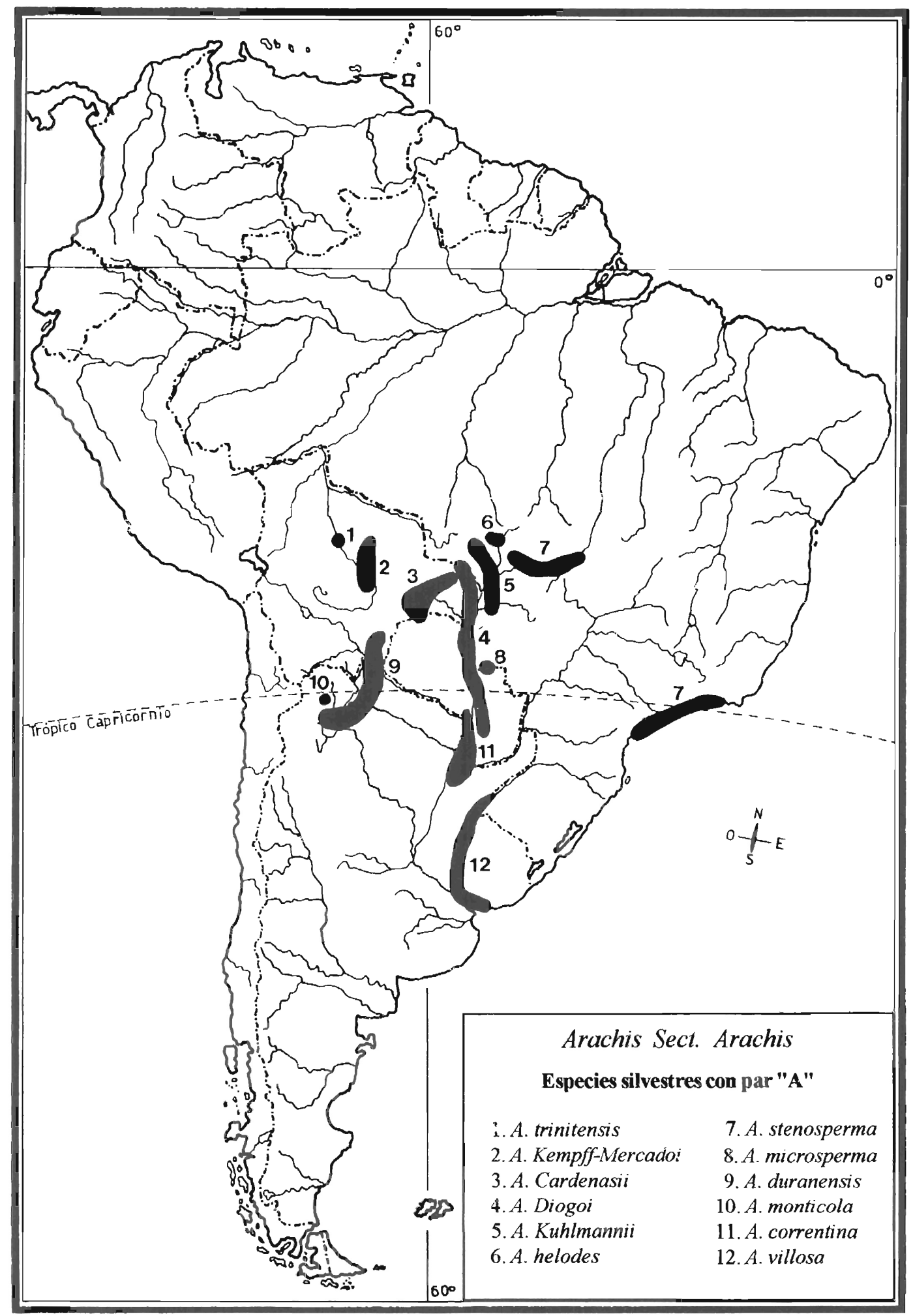

Fig. 4. Distribución geográfica de Arachıs Sect. Arachis. 


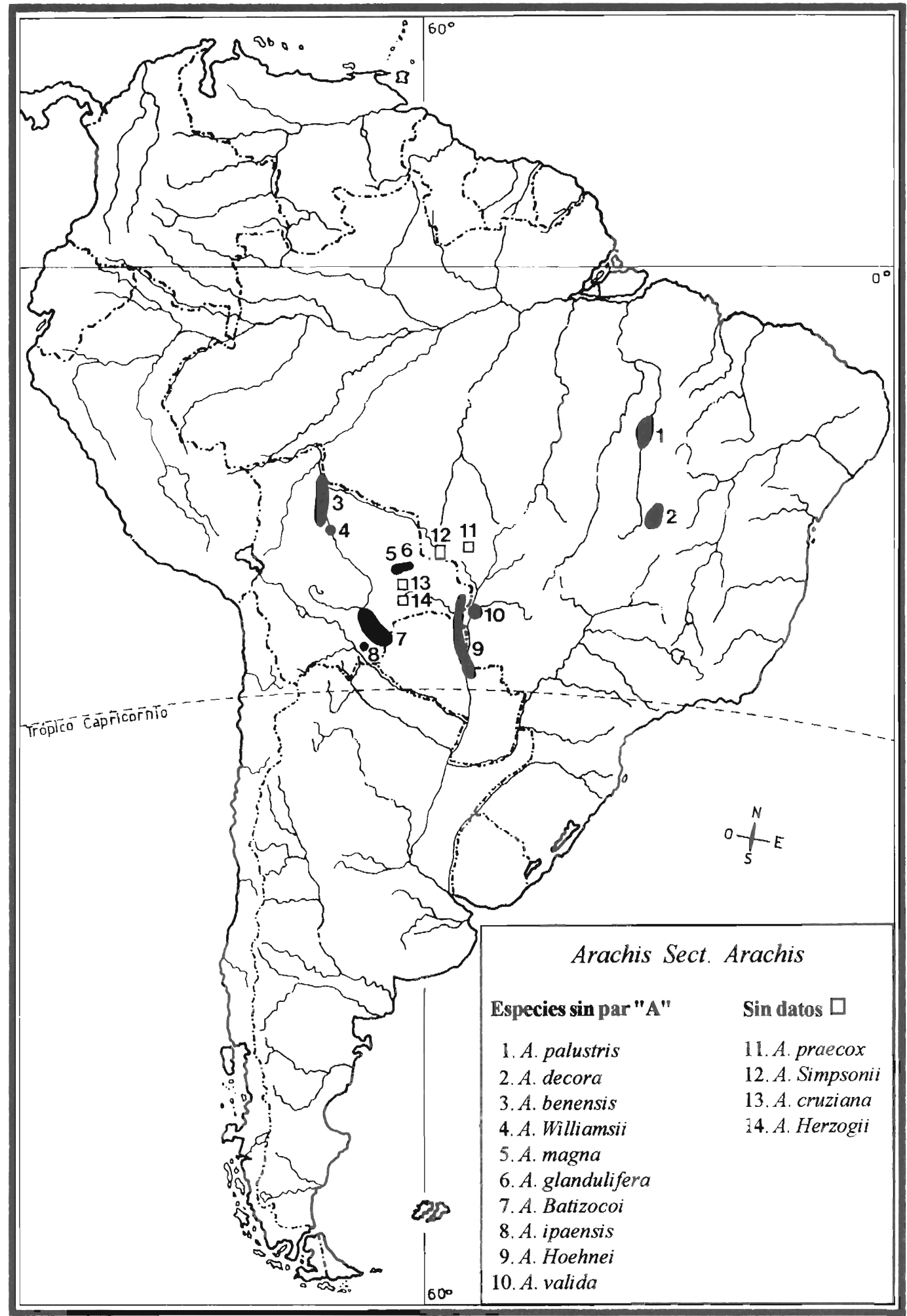

Fig. 5. Distribución geográfica de Arachis Sect. Arachis. 
al traslado por acción humana se presenta también en la costa atlántica (Krapovickas y Gregory, 1994).

En el origen del maní cultivado habrían intervenido 2 especies diploides, una de las cuales debería poseer cromosomas "A" y la otra carecer de ellos (Smartt et al., 1978; Femández y Krapovickas, 1994). Se constató que $A$. decora y $A$. palustris, las especies que ocurren más al norte (Fig. 5), no poseen dichos cromosomas; de este modo se estrecha aún más el área donde se habría producido la domesticación del maní. Pero, al mismo tiempo, el área posible es ampliada al noroeste, hasta Trunidad (Bolivia), ya que A. trinitensis (con par " $\mathrm{A}$ ") y A. Williamsii (sin par "A") son simpátricas.

\section{Agradecimientos}

Deseo expresar mi agradecimiento al Ing. Agr. A. Fernández por su constante apoyo, al Ing. Agr. A. Krapovickas por las sugerencias recibidas, al Dr. J.F.M. Valls (CENARGENEMBRAPA) y al Ing. Agr. E. Giandana (INTAEEA-Manfredi) por la gentileza en el envío de semillas. Al Sr. Víctor Maruñak por la confección de los mapas. Este trabajo fue llevado a cabo mediante el apoyo económico de las si- guientes instituciones: CONICET y SECYT de la UNNE.

\section{Bibliografía}

FERNANDEZ, A.\& A. KRAPOVICKAS. 1994. Cromosomas y evolución en Arachis (Leguminosae). Bonplandia 8(14).187-220.

GREGORY, W.C., M.P. GREGORY, A. KRAPOVICKAS, B.W. SMITH \& J.A. YARBROUGH. 1973. Structures and genetic resources of Peanuts, en C.T. Wilson (ed.). Peanuts-Culture and Uses. Am. Peanut Res. and Educ. Assoc, Stillwater, OK. Chapier 3:47-134.

HUSTED, L. 1933. Cytological Studies on the Peanut, Arachis. 1. Chromosome number and morphology. Cytologia 5:109-117.

HUSTED, L. 1936. Cytological Studies on the Peanut, Arachis. U. Chromosome number, morphology and their application to the problem of the origin of the cultivated forms. Cytologia 7:396-423.

KRAPOVICKAS, A. \& W.C. GREGORY. 1994. Taxonomía del género Arachis (Leguminosae). Bonplandia 8(1-4):1186.

KRAPOVICKAS A. \& V.A. RIGONI. 1951. Estudios citológicos en el género Arachis. Revista Invest. Agric. $5(3): 289-293$

KRAPOVICKAS, A. \& V.A. RIGONT. 1957. Nuevas especies de Arachss vinculadas al problema del origen del mani. Darwiniana 11 (3):431-458.

SMARTT, J.; W. C. GREGORY \& M. PFLUGE GREGORY. 1978. The genomes of Arachis hypogaea. 1. Cytogenetic studies of putative genome donors Euphytica 27:665675 